\title{
Evidence Of An Intraerythrocytic Secretory Pathway In Erythrocytes Infected With Plasmodium falciparum
}

\author{
Timothy Schneider, Megan E. O’Donnell and Theodore F. Taraschi \\ Department of Pathology, Anatomy and Cell Biology, Jefferson Medical College, Philadelphia, \\ PA 19107, USA
}

During the development of the asexual stage of the malaria parasite, Plasmodium falciparum $(P f)$, the composition, structure and function of the host cell membrane is dramatically altered, including the ability to adhere to vascular endothelium. Crucial to these changes is the transport of parasite proteins, which become associated with or inserted into the erythrocyte membrane. Protein and membrane targeting beyond the parasite plasma membrane (PPM) must require unique pathways, given the parasites intracellular location within a parasitophorous vacuolar membrane (PVM) and the lack of organelles and biosynthetic machinery in the host cell necessary to support a secretory system. It is not clear how these proteins cross the PVM or how they traverse the erythrocyte cytosol to reach their final destinations.

The identification of 1) Pf homologues of the proteins Sar1p and Sec31p, which are essential components of the COPII-based secretory system in higher eukaryotes and 2) a Pf $N$-Ethylmaleimide-sensitive factor (NSF), which plays a central role in vesicular trafficking in eukaryotic cells in combination with 3) the observation of electron-dense, possibly coated, secretory vesicles bearing the parasite proteins PfEMP1 and PfEMP3 in the host cell cytosol of $P$. falciparum infected erythrocytes ${ }^{1-3}$ provided the first direct evidence of a vesicle-mediated pathway for the trafficking of some parasite proteins to the erythrocyte membrane.

The major advance in uncovering the parasite-induced secretory pathway was made by incubating IRBC with aluminum tetrafluoride, an activator of GTP-binding proteins, which resulted in the accumulation of the vesicles into multiple vesicle strings (Figure 1). These vesicle complexes were often associated with and closely abutted the erythrocyte membrane, but were apparently prevented from fusing by the aluminum fluoride treatment, making their capture by electron microscopy possible. Immunoelectron microscopy revealed that PfEMP1 was associated with the coated vesicles in the erythrocyte cytosol. It appears that malaria parasites export proteins into the host cell cytosol to support a vesicle-mediated secretory pathway.

References

[1] T.F. Taraschi et al., (2001) Int. J. Parasitol.31, 1381.

[2] D.P. Trelka et al., (2000) Mol. Biochem. Parasitol. 106, (2000).

[3] J.A. Gormley et al., (1992) J. Cell Biol. 119, 1481. 

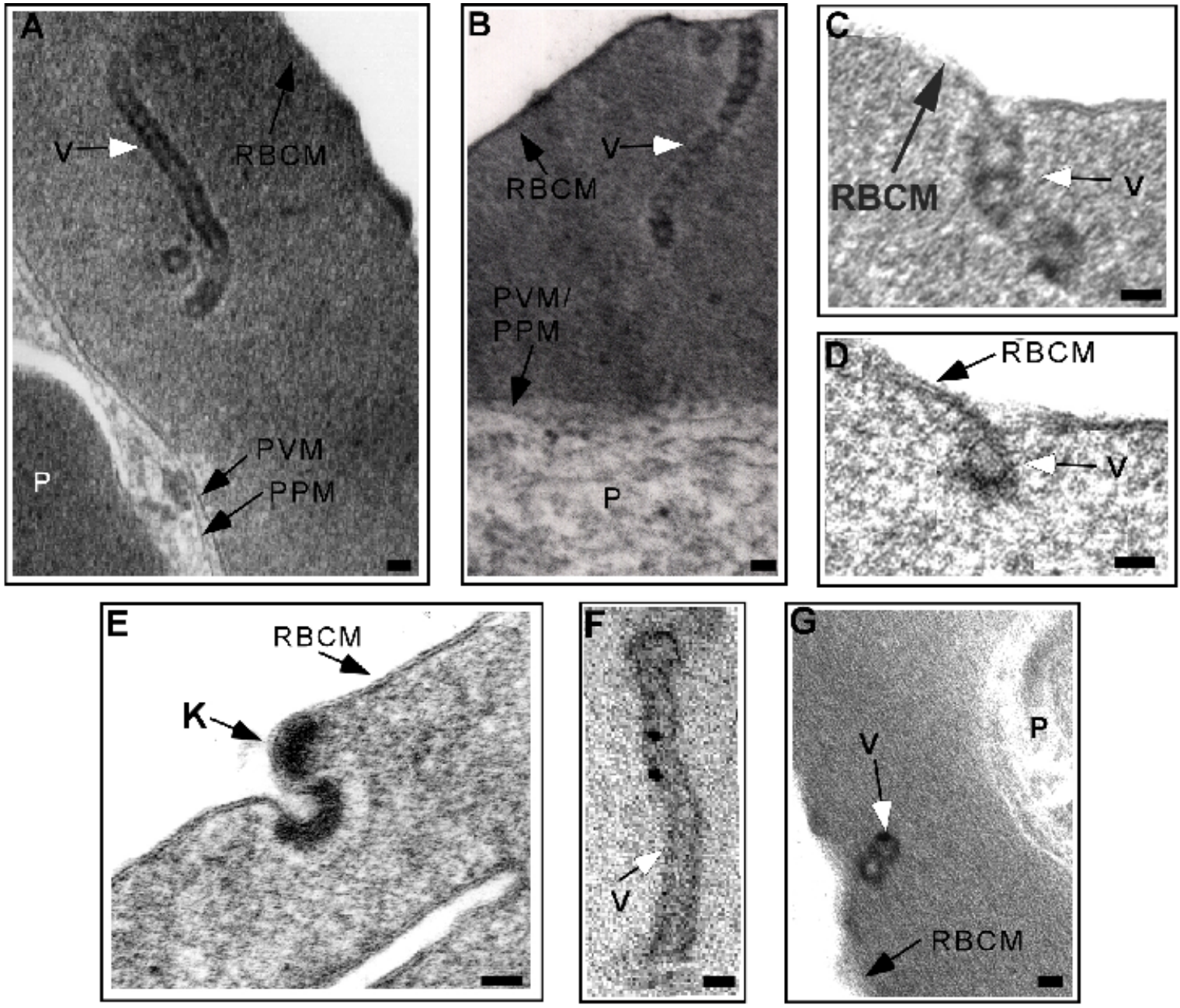

Figure 1. Treatment of Erythrocytes Infected with the Human Malaria Parasite Plasmodium falciparum Reveals an Intraerythrocytic Secretory Pathway. Mature parasites treated for $1 \mathrm{~h}$ with aluminum fluoride (AlF4), an activator of $G$-proteins revealed A) strings of coated vesicles in the red blood cell (RBC) cytosol (white arrow) and also B) extending to the RBC membrane (white arrow). In some sections C) strings of vesicles and D) single vesicles appeared to be in the process of uncoating and fusing with the RBCM. Electron dense knobs on the surface of the RBCM which are involved in cytoadherence of infected erythrocytes to vascular endothelium, appear to arise from the fusion of coated vesicles with the RBCM. The components of the electron translucent halo around the fusing coated vesicle in E) are unknown. Mature parasites were processed for immunoelectron microscopy and probed with a rabbit anti-Plasmodium falciparum erythrocyte membrane protein 1 (PfEMP1) IgG followed by a goat anti-rabbit IgG coupled to $15 \mathrm{~nm}$ gold. PfEMP1 is distributed on F) electron dense vesicles arranged in strings within the erythrocyte cytosol and $G$ ) with dense vesicles that appear to fuse with the RBCM. Scale bar $=70 \mathrm{nM}$. 